\title{
Fremian
}

Fermilab-TM-2451-APC-TD 2010

\section{Radiation Shielding for the Fermilab Vertical Cavity Test Facility*}

\author{
Camille Ginsburg and Igor Rakhno \\ Fermilab, P.O. Box 500, Batavia, IL 60510
}

March 17, 2010

\begin{abstract}
The results of radiation shielding studies for the vertical test cryostat VTS1 at Fermilab performed with the codes FISHPACT and MARS15 are presented and discussed. The analysis is focused on operations with two RF cavities in the cryostat.
\end{abstract}

\section{Introduction}

The vertical cavity test facility (VCTF) for superconducting RF cavities in Industrial Building 1 at Fermilab has been in operation since 2007. The facility currently consists of a single vertical test cryostat VTS1. Radiation shielding for VTS1 was designed for operations with single 9-cell $1.3 \mathrm{GHz}$ cavities, and the shielding calculations were performed using a simplified model of field emission as the radiation source [1,2]. The operations are proposed to be extended in such a way that two RF cavities will be in VTS1 at a time, one above the other, with tests for each cavity performed sequentially. In such a case the radiation emitted during the tests from the lower cavity can, in part, bypass the initially designed shielding which can lead to a higher dose in the building. Space for additional shielding, either internal or external to VTS1, is limited. Therefore, a re-evaluation of the radiation shielding was performed.

An essential part of the present analysis is in using realistic models for cavity geometry and spatial, angular and energy distributions of field-emitted electrons inside the cavities. The calculations were performed with the computer codes FISHPACT [3] and MARS15 [4].

\footnotetext{
${ }^{*}$ Work supported by Fermi Research Alliance, LLC, under contract DE-AC02-07CH11359 with the U. S. Department of Energy.
} 


\section{Geometry Model}

A full cross section and fragment of the developed three-dimensional model of the test facility are shown in Figs. 1 and 2. As for the color scheme employed to denote materials in the model, the following convention applies: white, light blue, gray and red colors correspond to vacuum, air, regular concrete and stainless steel, respectively. In addition, in this model the violet and brown colors correspond to lead and borated polyethylene, respectively. The internal shielding itself consists of two cylindrical lead blocks and cylindrical layers of steel and borated polyethylene immediately above the upper RF cavity. Other technological components of the facility also serve as an extra shielding: (i) layers of copper and G10 above the internal shielding and under the top plate; (ii) the steel top plate; (iii) several cylindrical shells around the RF cavities-magnetic shield of Cryoperm 10 with aluminum support liner, helium vessel made of copper, and steel vessel.

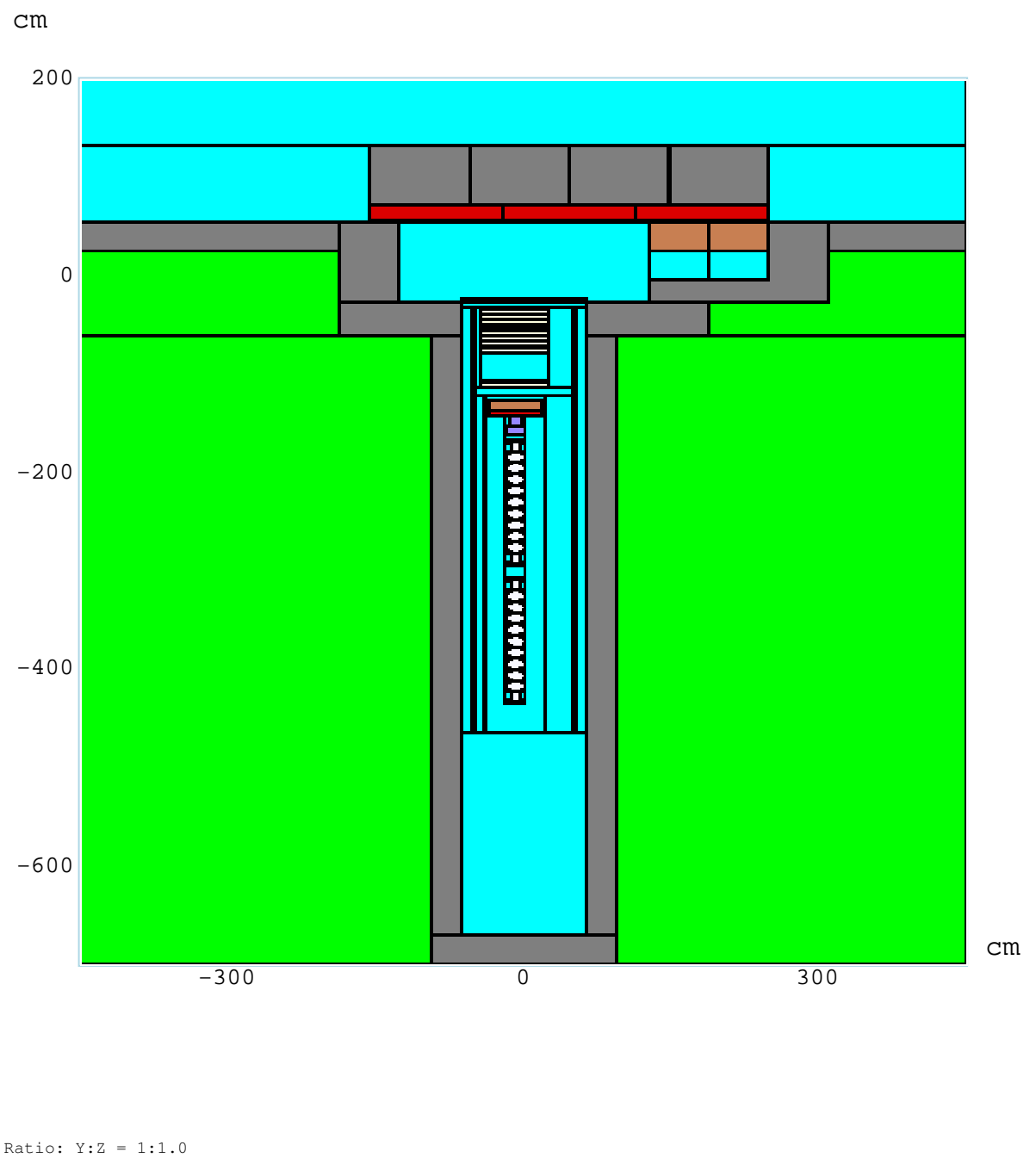

Figure 1: A full cross section of the MARS15 model of the facility with two RF cavities. 
Various small components of the facility like numerous cables and pipes are not included in the computer model. For technological reasons, there is a small offset of the vertical axis of the two RF cavities and the internal shielding to the left relative to the central axis of the cryostat, as shown in the Figures. The boundaries between different regions are shown with black lines. It should be noted also that, when the resolution of a figure is inadequate to show small regions, these regions appear as black ones.

The realistic geometry model of the superconducting RF cavities was taken from the TESLA cavity design [5].
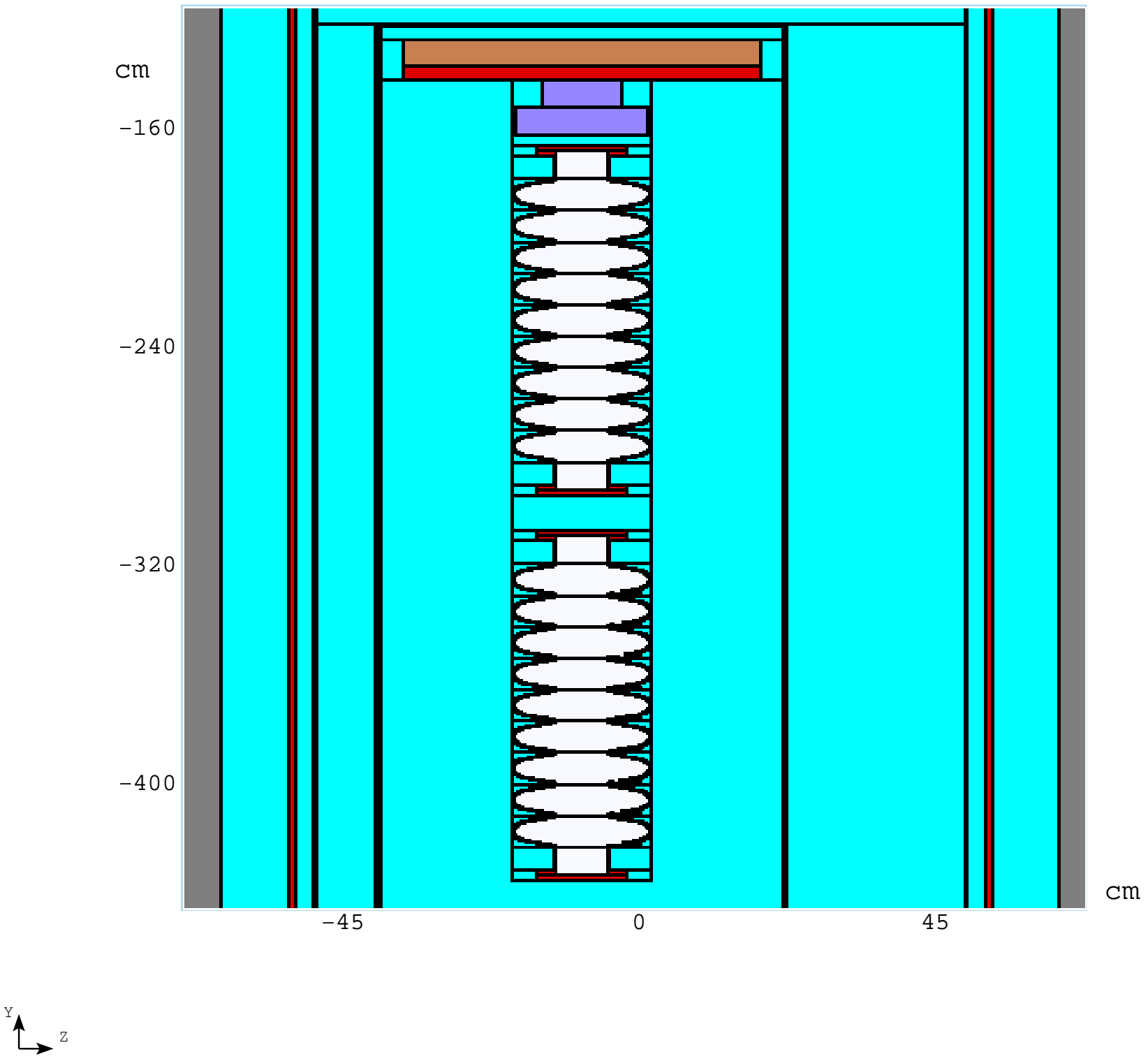

Aspect Ratio: $Y: Z=1: 0.41361$

Figure 2: A fragment of the MARS15 model of the facility with two RF cavities. 


\section{Source term}

A realistic model for the radiation source term was developed to describe the trajectories and energy distributions of field emitted electrons generated in RF cavities at high accelerating gradients. The FISHPACT code has been used to model electrons emitted from sites in the vicinity of cavity irises, where the surface electric field is highest. FISHPACT is interfaced with POISSON SUPERFISH [6], a simulation package used to calculate RF electromagnetic fields in two dimensions. Although the simulation provides a field emission current, given input field emission parameters, only the electron trjectories and energies have been used here. The dose estimated in the simulation using parameters from literature [7], was found to be substantially higher than for existing data, so data have been used to normalize the dose, as described in Section 4.

The cavity cell structure and the surface electric field from simulation are shown as a function of cavity $\mathrm{Z}$ in Fig. 3. Electrons emitted from the iris region are most likely to be accelerated along the cavity axis and acquire significant energy. The trajectory and energy depend on the RF phase of the cavity. An example of simulated trajectories for an emission site near an iris in which electrons can reach an energy almost as high as the cavity accelerating gradient is shown in Fig. 4. The simulation is two-dimensional, and should be understood to be symmetric around the $\mathrm{Z}$ axis.

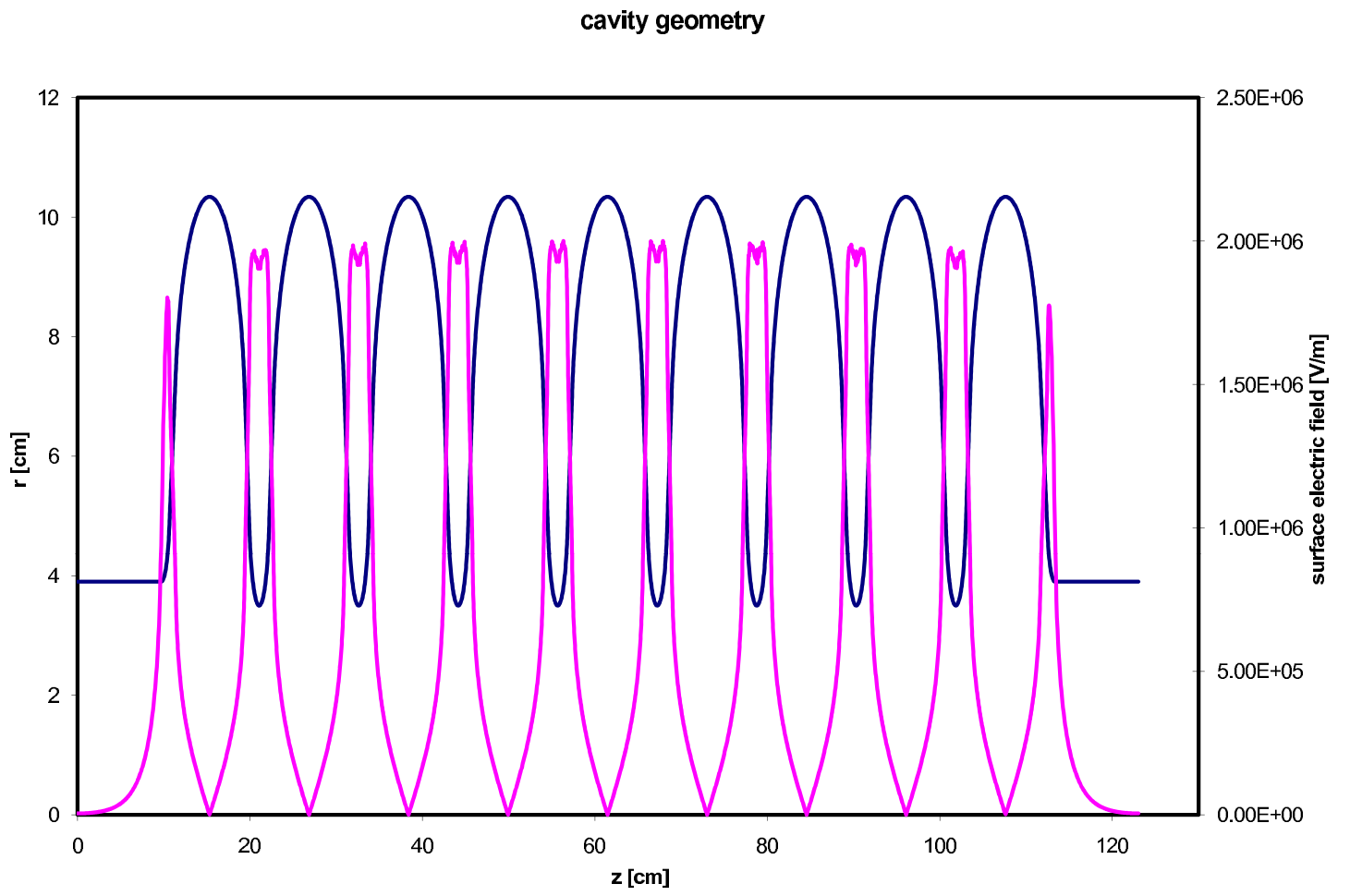

Figure 3: The cavity electric field (pink, normalized to $1 \mathrm{MV} / \mathrm{m}$ ), and the cavity cell structure (blue, $\mathrm{cm}$ ) as a function of cavity $\mathrm{Z}$ from a SUPERFISH simulation. Note that the electric field peaks in the cavity iris regions. 


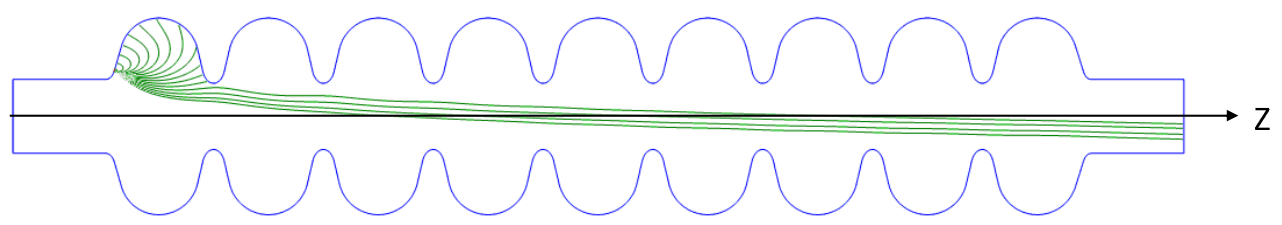

Figure 4: Simulated electron trajectories generated in a 9-cell SRF cavity with an accelerating gradient of $30 \mathrm{MV} / \mathrm{m}$. The green curves correspond to electron trajectories for 10 degree increments in the RF phase, for the half period in which the electric field has the correct sign to pull electrons from the surface.

The maximum gradient of $30 \mathrm{MV} / \mathrm{m}$ has been chosen to correspond to the largest gradient at which field emission is likely to result in a substantial x-ray flux at the cryostat top plate, as determined from data. In addition, this is approximately the largest gradient for which the facility must be able to test typical cavities without interrupting the RF system because of trips by the radiation monitoring system.

\section{Normalization of calculated dose rates}

Normalization of the calculated dose distributions was performed using the measured dose rates for similar RF cavities obtained from the DESY/TTF vertical test facility. The procedure is described in detail in $[1,2]$.

\section{Calculated dose rate distributions around the facility}

The calculated distributions of prompt dose rate around the facility are shown in Fig. 5. For the case of the upper tested cavity the predicted dose is well below $1 \mathrm{mrem} / \mathrm{hr}$. It means that the initial shielding design, developed for a single tested cavity and based on an approximate source of field emission, is very conservative when compared to the realistic model of the field emission. Note that, as of the time of this writing, the radiation monitors have never tripped the RF system because of high dose rate. In fact, only slightly elevated levels with respect to background have ever been measured at VCTF, even for those cavities with significant field emission at high gradient.

For the lower tested cavity the predicted dose above the removable shielding block is higher and the hottest spot is about $6 \mathrm{mrem} / \mathrm{hr}$. The radiation is comprised of mostly gammas, and the corresponding calculated energy spectrum is shown in Fig. 6. The average energy in the spectrum is about $2 \mathrm{MeV}$ and several millimeters of steel can reduce the dose in the hottest spot down to $5 \mathrm{mrem} / \mathrm{hr}$. Taking into account the presence of numerous cables and pipes on the top plate, one can state that the extra few millimeters of steel shielding are effectively installed. 

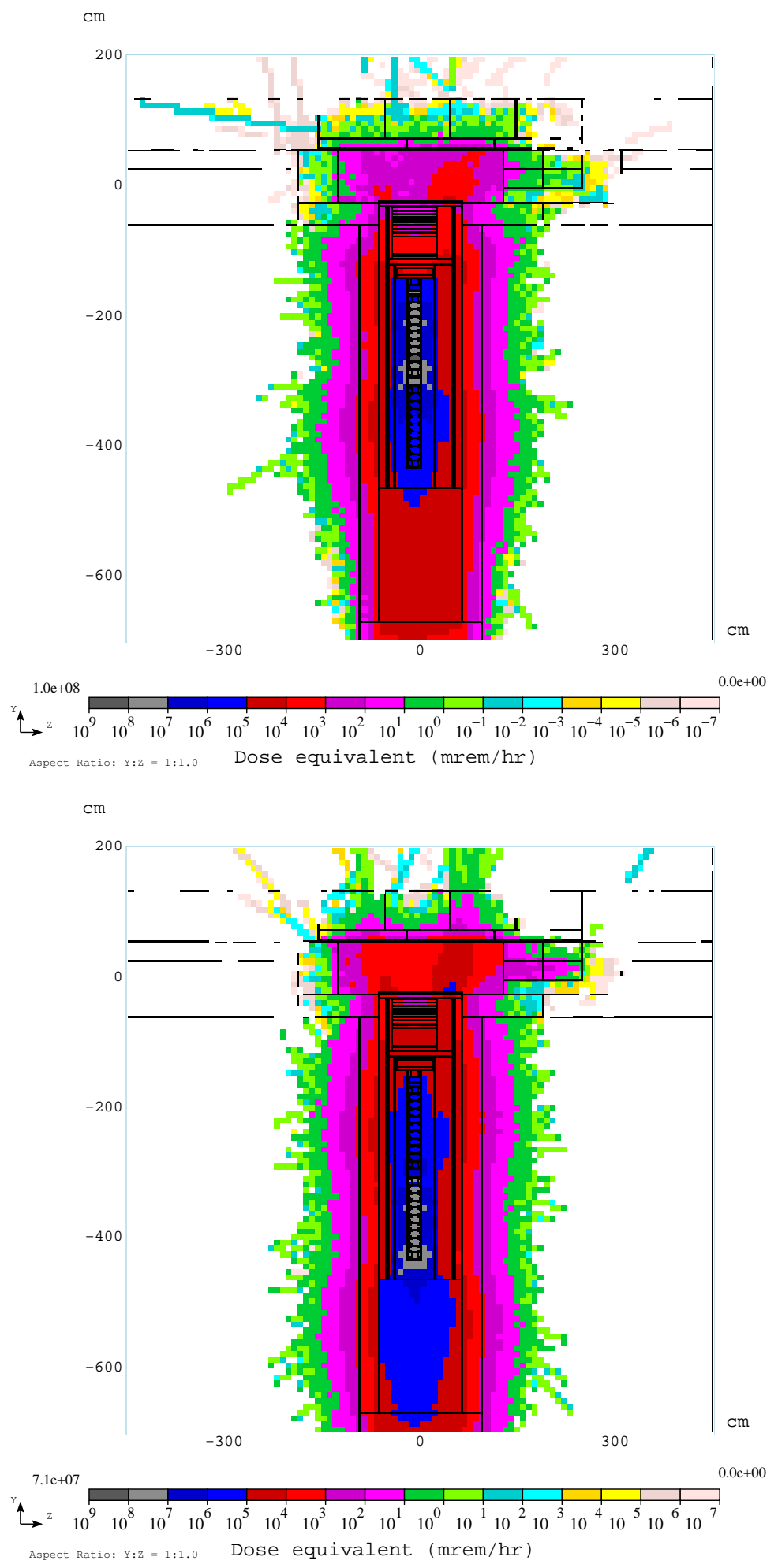

Figure 5: The calculated distributions of prompt dose rate around the test facility with the upper (top) and lower (bottom) RF cavity being tested. 


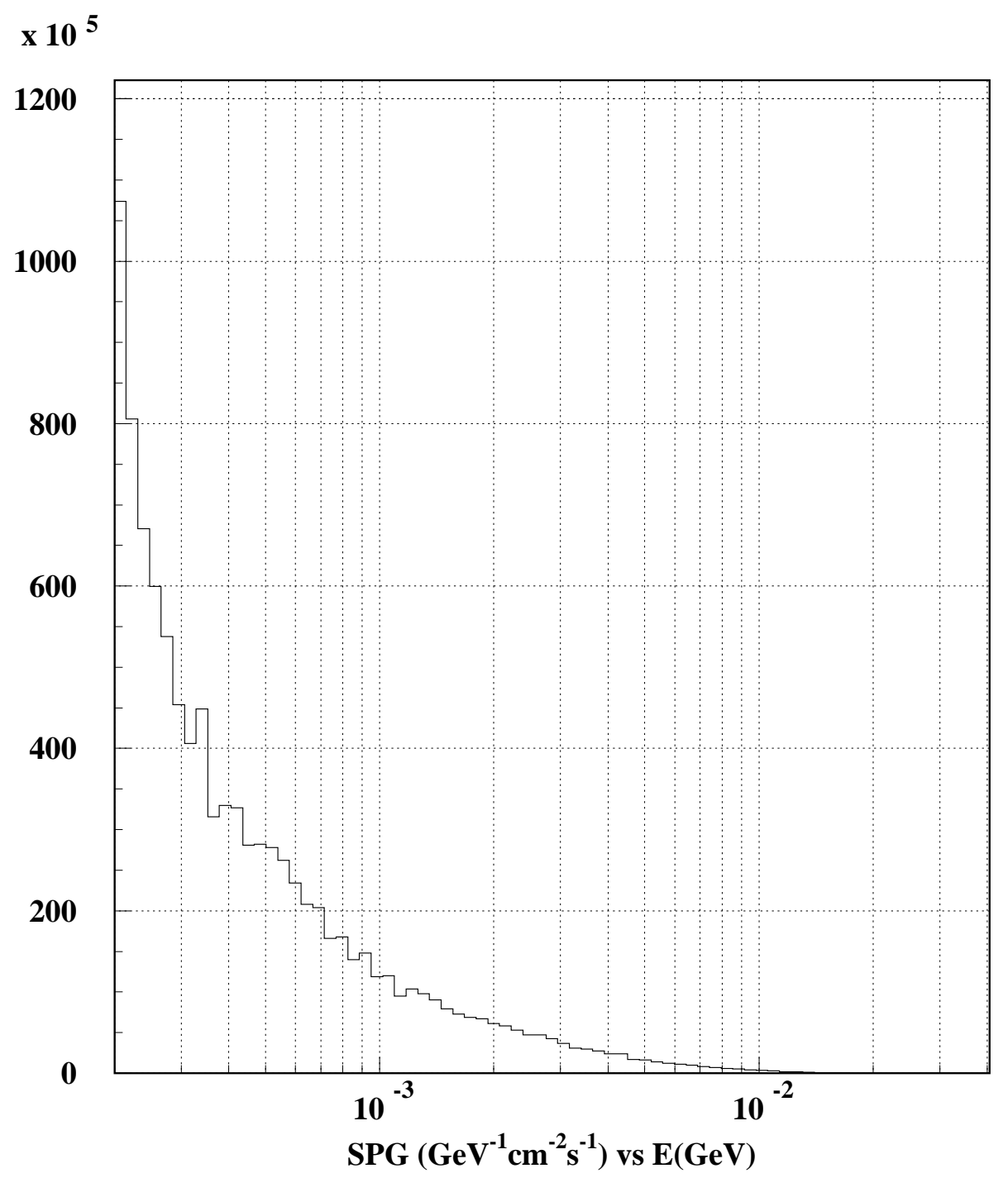

Figure 6: The calculated energy spectrum of gammas in the region between the steel top plate and removable concrete block for the case of the lower RF cavity being tested. The normalization is arbitrary.

\section{Conclusions}

Radiation shielding studies have been performed for the vertical test cryostat VTS1 at Fermilab for the configuration in which two RF cavities are in the cryostat and tested serially. These studies were performed using an improved model of the radiation source. The resulting radiation distributions are presented. 


\section{References}

[1] I. Rakhno, "Radiation Shielding Study for Superconducting RF Cavity Test Facility at Fermilab," Fermilab-TM-2350-AD (2006).

[2] I. Rakhno, "Radiation Shielding Issues for Superconducting RF Cavity Test Facility at Fermilab," Fermilab-TM-2367-AD (2006).

[3] E. Donoghue, G. Wu, J. Mammosser, R. Rimmer, M. Stirbert, L. Phillips and H. Wang, "Studies of electron activities in SNS-type superconducting RF cavities," Proc. SRF2005 Conference, Ithaca, New York, June 2005, TuP67.

[4] N.V. Mokhov, “The MARS code system user's guide version 13(95)," FermilabFN-628 (1995); N.V. Mokhov, S.I. Striganov, "MARS15 overview," Proc. Hadronic Shower Simulation Workshop, Batavia, Illinois, USA, 6-8 September, 2006, Vol. 896, pp. 50-60, American Institute of Physics, Melville, NY (2007); http://www-ap.fnal.gov/MARS/.

[5] B. Aune et al., Phys. Rev. STAB 3, 092001 (2000).

[6] K. Halbach and R. F. Holsinger, "SUPERFISH - A Computer Program for Evaluation of RF Cavities with Cylindrical Symmetry," Particle Accelerators 7 (1976) 213-222; http://laacg1.lanl.gov/laacg/services/download_sf.phtml.

[7] H. Padamsee, J. Knobloch, and T. Hays, RF Superconductivity for Accelerators, 2nd ed. (Wiley, Weinheim, 2008) pp.230-242. 